\title{
Flexible fiber in interaction with a dense granular flow close to the jamming transition
}

\author{
Nicolas Algarra ${ }^{1, \star}$, Marguerite Leang ${ }^{1}$, Arnaud Lazarus ${ }^{2}$, Damien Vandembroucq ${ }^{1}$, and Evelyne Kolb ${ }^{1, \star \star}$ \\ ${ }^{1}$ PMMH, CNRS UMR 7636, ESPCI Paris, UPMC Univ Paris 06 and Univ Paris 07, 10 rue Vauquelin, 75005 Paris, France \\ ${ }^{2}$ Sorbonne Universités, UPMC Univ Paris 06, CNRS, UMR 7190, Institut Jean Le Rond d'Alembert, 75005, Paris, France
}

\begin{abstract}
We propose a new fluid/structure interaction in the unusual case of a dense granular medium flowing against an elastic fiber acting as a flexible intruder. We study experimentally the reconfiguration and the forces exerted on the flexible fiber produced by the flow at a constant and low velocity of a two-dimensional disordered packing of grains close but below the jamming transition.
\end{abstract}

\section{Introduction}

The study of fluid-structure interaction has been traditionally developed in the context of mechanics and engineering for optimizing structures against wind or water flow. Recently there have been fundamental works dealing with the drag reduction and reconfiguration of flexible objects into streamlined shapes in controlled water flows [1], wind tunnels [2] or into 2D soap film flows [3]. This field has been extended to bio-physical domains, like the reconfiguration of plants and leaves under wind or of algae in marine flows [4]. In animals, the flexible nature of the body, wings or fins interacting with the external medium could be of particular interest for optimizing the thrust efficiency, like for the swimming of fishes or eels, the flying of birds and insects [5], the crawling of snakes and the diving of sandfish lizards [6] or the digging of worms in mud sediments [7]. Even at a smaller scale, the swimming of microorganisms with flagella or even the beating of ciliary flagella [8] take advantage of the flexibility.

In the present work, we propose a new fluid/structure interaction in the unusual case of a 2D dense granular medium flowing against an elastic fiber acting as a flexible intruder: One tip of the fiber is clamped while the other is free and initially faces the granular flow, as if the fiber was penetrating the granular medium. The mechanical resistance produced by the cluster of grains contacting the fiber tends to bend or buckle the flexible intruder, which in turn modifies the granular flow.

More precisely, we study experimentally the reconfiguration and the forces exerted on an elastic mylar beam (the fiber) produced by the flow at a constant and low velocity of a two-dimensional, bidisperse, dense and disordered packing of metallic cylinders (the granular fluid). Imposing the fiber geometry like its length or thickness

\footnotetext{
${ }^{\star}$ e-mail: nicolas.algarra@espci.fr

$\star \star$ e-mail: evelyne.kolb@upmc.fr
}

sets the critical buckling force the fiber is able to resist if it was not supported by lateral grains, while increasing the granular packing fraction might laterally consolidate the fiber and prevent it from buckling. But on the other side, the approach to jamming transition by increasing the granular packing fraction will be characterized by a dramatically increasing size of the cluster of connected grains forming a solid block acting against the fiber, which might promote the fiber deflection. Thus, we investigated the fiber deflexion as well as the forces experienced by the fiber and compared them with theoretical predictions from Elastica for particular models of loadings along the fiber.

\section{Experimental setup}

The granular medium is an horizontal assembly of around 6800 rigid cylinders of external diameters $d_{1}=4 \mathrm{~mm}$ (in number $N_{1}$ ) and $d_{2}=5 \mathrm{~mm}$ (in number $N_{2}=\frac{4}{7} N_{1}$ ) and height $h=3 \mathrm{~mm}$. The grains lie on a rectangular glass plate closed by rigid walls (cell width $W \approx 54 d_{2}$ and maximum cell length $D \approx 94 d_{2}$ ) and are moved at a constant velocity $V_{0}=5 / 6 \mathrm{~mm} / \mathrm{s}$ corresponding to a quasi-static regime for granular flows. The initial packing fraction of grains $\phi_{0}$ can be adjusted by slightly shifting one of the wall along the cell length, such that $\phi_{0}$ is in the range $80 \%$ $83 \%$, close to but below the 2D (bidimensional) jamming transition at $83.56 \%$ observed in our similar preceding experiments performed on rigid intruders [9],[10]. The fiber is a mylar beam of rectangular cross-section with a thickness $t=350 \mu \mathrm{m}$, a height $h=3 \mathrm{~mm}$ and a length $L$ which is fixed in a range of 2 to 10 diameters of large grains $d_{2}$. One tip of the fiber is clamped and connected to a 6-axis force and torque sensor mounted slightly above the horizontal plate. The other fiber extremity is free. The fiber is initially aligned along the $y$-axis such that the free extremity faces the granular flow, as if the fiber was penetrating the granular medium. A CCD camera of $1600 \times 1200$ pixels 
placed above the set-up records images at a frequency of $2 \mathrm{~Hz}$, corresponding to a plate displacement (along the $\mathrm{y}$ axis) of $U_{0}=5 / 12 \mathrm{~mm}=d_{2} / 12$ between 2 successive images (see Fig. 1). Ten similar experiments are performed for each set of parameters $L$ and $\phi_{0}$.

\section{Results}

From the series of images obtained for fiber lengths $L \geq$ $2.5 \mathrm{~cm}=5 d_{2}$, we observe that the fiber experiences three successive regimes during its penetration relative to the granular medium. In a first regime (I), the fiber slightly fluctuates around its initial straight position (see Fig. 1a for example for $L=3 \mathrm{~cm}, \phi_{0}=80.85 \%$ ). After a traveling distance $\ell$ that depends on the initial organization of grains, the fiber irremediably inclines itself towards one side, either right or left, and enters a regime of irreversible bending (Regime II) (Fig.1b), i.e. the fiber can not come back to its straight position. We also observe the formation of a cavity empty of grains behind the fiber. The area of the cavity increases while the fiber continues to bend further. Once the main part of the fiber is placed perpendicular to the arrival flux of grains, we define a third regime (III) called avalanche regime: successive events of loading occur when the fiber bends further (Fig.1c), followed by rapid unloading events where the fiber comes back (Fig.1d). These unloadings are associated with notable grains recirculations from upstream to downstream around the free end of the fiber. For fiber lengths $L<2.5 \mathrm{~cm}$ and $\phi_{0}<82.0 \%$, only the first regime of small fluctuation (I) is observed and in average, the fiber stays straight over the whole penetration distance. The threshold for straight penetration is obtained for slightly smaller lengths $\left(L<2.0 \mathrm{~cm}=4 d_{2}\right)$ when $\phi_{0} \geq 82.0 \%$ approaches the jamming transition.

\subsection{Fiber deflection}

From each image we extract the fiber shape. Then we compute the lateral deflection (along the $\mathrm{x}$-axis) of the fiber free end $\delta$ as a function of the penetration distance $\ell$ in the granular medium. As shown in Fig.2, the three regimes are clearly visible and the deflections corresponding to the images of Fig. 1 are indicated with letters. In regime I, the value of $\delta$ oscillates around zero without exceeding one grain size. Then during regime II, the deflection increases with some fluctuations but without returning to zero. The limit between regime I and II is defined in the following way: On Fig. 2, we determine the location of the last passage of the deflection $\delta$ through zero, corresponding to the last time when the fiber was straight. Then we identify the maximum $\delta_{1}$ of the amplitude of deflection before this point. This is done for each experiment performed with the same control parameters $L$ and $\phi_{0}$, which allows determining a maximum deflection in the regime I equals to $\delta_{1 \text { Max }}=0.29 d_{2}=0.48 L$ for $L=3 \mathrm{~cm}$ and $\phi_{0}=80.85 \%$ in Fig. 2. Note that the typical deflection at the transition I-II is always of the order of one grain size. The deflection dramatically increases during regime II, which corresponds

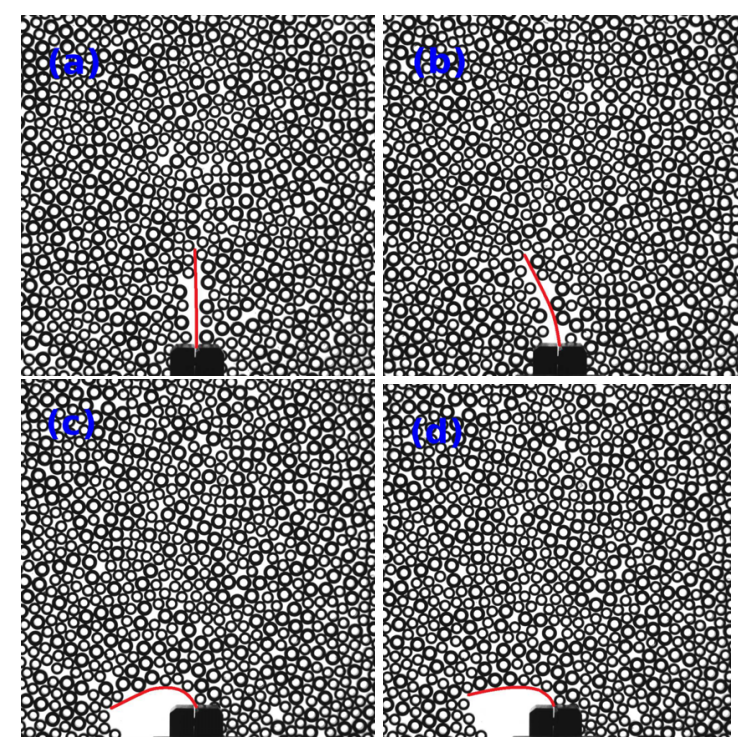

Figure 1. Images of the fiber of length $L=3 \mathrm{~cm}$ and thickness $t=350 \mu m$ inside a 2D granular material of initial packing fraction $\phi_{0}=80.85 \%$. (a) After a relative displacement of $\ell=2 d_{2}$ (Regime I). (b) $\ell=10.16 d_{2}$ (Regime II). (c) Image just before an unloading event at $\ell=34.83 d_{2}$ (Regime III) (d) Following image just after the unloading (avalanche) event at $\ell=35 d_{2}$.

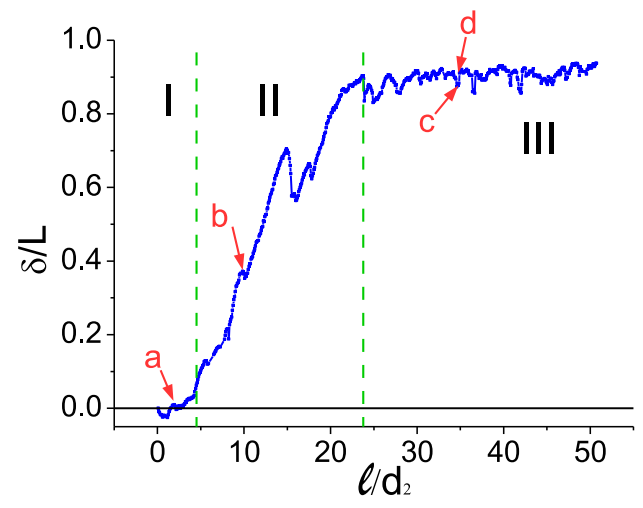

Figure 2. Lateral deflection of the fiber free end $\delta$ normalized by the fiber length $L$ as a function of the distance of penetration $\ell$ (normalized by the grain diameter $d_{2}$ ) for the experiment shown in Fig. $1\left(L=3 \mathrm{~cm}, \phi_{0}=80.85 \%\right.$ and $\left.t=350 \mu \mathrm{m}\right)$. The numbers I, II, III correspond to the three successive regimes, and the letters $\mathrm{a}, \mathrm{b}, \mathrm{c}$ et $\mathrm{d}$ to the corresponding labelled images of Fig.1.

to the fiber bending aside. Then the value of $\delta$ saturates in Fig. 2 for $L=3 \mathrm{~cm}$ when the regime III is obtained. For longer lengths, there is no clear plateau as the fiber continues to bend further with a pronounced hook shape. But we observed large fluctuations corresponding to numerous loading-unloading events characteristic of the last regime. In all cases, the limit between regime II and III is defined as the average over the 10 experiments of the maximum deflection. It is obtained at $\delta=0.90 L$ for the Fig. 2 . 

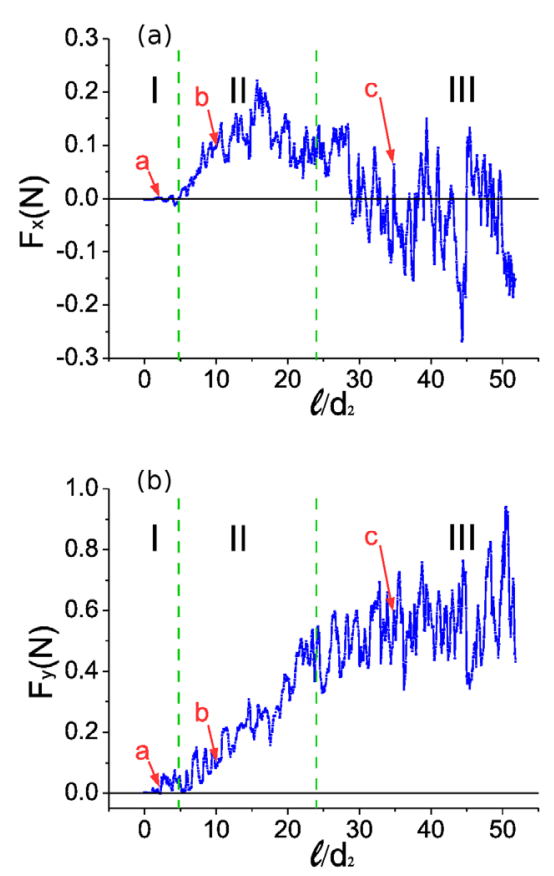

Figure 3. Evolution with the penetration distance $\ell$ of the components of forces detected by the force sensor at the fiber clamping point for the individual experiment of Fig. 1 with $L=3 \mathrm{~cm}$, $\phi_{0}=80.85 \%$ et $t=350 \mu \mathrm{m}$. The zones I, II, III correspond to the ones defined in Fig. 2 as well as the images a, b et c. (a) Cross-flow force $F_{x}$. (b) Drag force $F_{y}$.

\subsection{Drag force}

With the sensor located at the clamping point, we also record the resultant of forces acting on the fiber: the drag force $F_{y}$ opposing the grains flux as well as the cross-flow force $F_{x}$. Both components are plotted as a function of the travelling distance $\ell$ (Fig. 3) and the limits of the three different regimes obtained in Fig. 2 are also reported on this graph. In the first regime (I), the cross-flow force $F_{x}$ is near zero, as the fiber stays almost straight. The drag force $F_{y}$ is small but always positive, as the fiber faces the arrival of grains. In the second regime (II), the fiber bends irreversibly on one side, leading to a rather regular increase of the drag force $F_{y}$ with the penetration distance $\ell$ till a value of around $0.5 \mathrm{~N}$. At the same time, we observe a positive component of the cross-flow force $F_{x}$ related to the deflection (Fig. 1b). In the third regime (III), the drag force $F_{y}$ seems to saturate although highly fluctuating, while the cross-flow force $F_{x}$ becomes negative. This is due to the hook shape of the fiber in this regime: The grains in contact with the fiber near its free extremity push the fiber in the reverse direction (towards the right side) compared with the grains near the clamping point that push the fiber to the left side.

As the evolution of forces and deflections seems to behave in a similar way, we plot them together in Fig. 4. Again the distinction between the three regimes is clearly visible. Of particular interest are the loop shapes in regime III, that correspond to temporal cycles of loading and unloading.
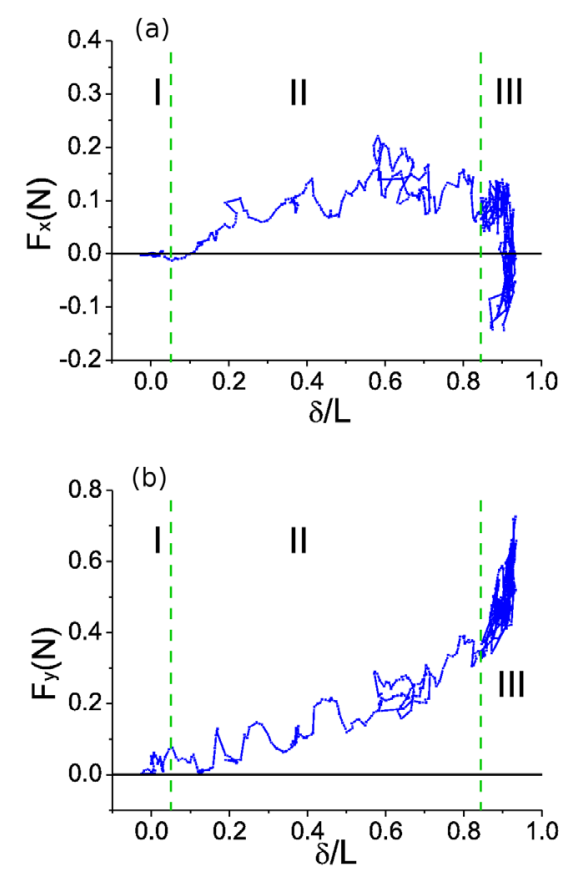

Figure 4. Forces measured at the fiber clamping point as a function of the lateral deflection $\delta$ obtained from the images and normalized by the fiber length $L=3 \mathrm{~cm}$ for $\phi_{0}=80.85 \%$.(a) Crossflow force $F_{x}$. (b) Drag force $F_{y}$.

\section{Comparison with simulations}

For the 10 experiments performed with the same control parameters $L$ and $\phi_{0}$, we obtained the forces on the fiber as well as the successive fiber shapes and corresponding lateral deflections. When plotting the cross-flow force (Fig. 5a) and drag force (Fig. 5b) as a function of the deflection, we observe a reasonable collapse of all the data provided by the 10 experiments. This suggests a generic mechanism of loading for the bending of the fiber observed in regime II. Moreover the presence of a cavity behind the fiber indicates that the fiber is loaded on its upstream side. Finally, as the grains contact the fiber along their height and as the corresponding friction is low, we propose to compare our experimental results with Elastica simulation of a fiber deflected by an homogenous orthogonal loading (Fig. 6). Elastica theory provides a way to treat large scale elastic deflections of beams [11].

Numerical results are reported in Fig. 5. They are obtained without any adjustable parameter and only depend on the mechanical and geometrical properties of the fiber (Young modulus $E=3.8 \mathrm{GPa}$ and Poisson ratio $v=0.4$ ). This simple model of loading gives a reasonable agreement for capturing the envelopes of Fig.5 for the experiments for $L=3 \mathrm{~cm}$. This loading is associated to the cluster of grains that piles up on the upstream side of the fiber. For better comparison with the simulation, the same force data provided by the 10 similar experiments have been averaged inside windows of $\delta / L$ of width 0.05 (Fig.7). The error bars correspond to the standard deviation. 

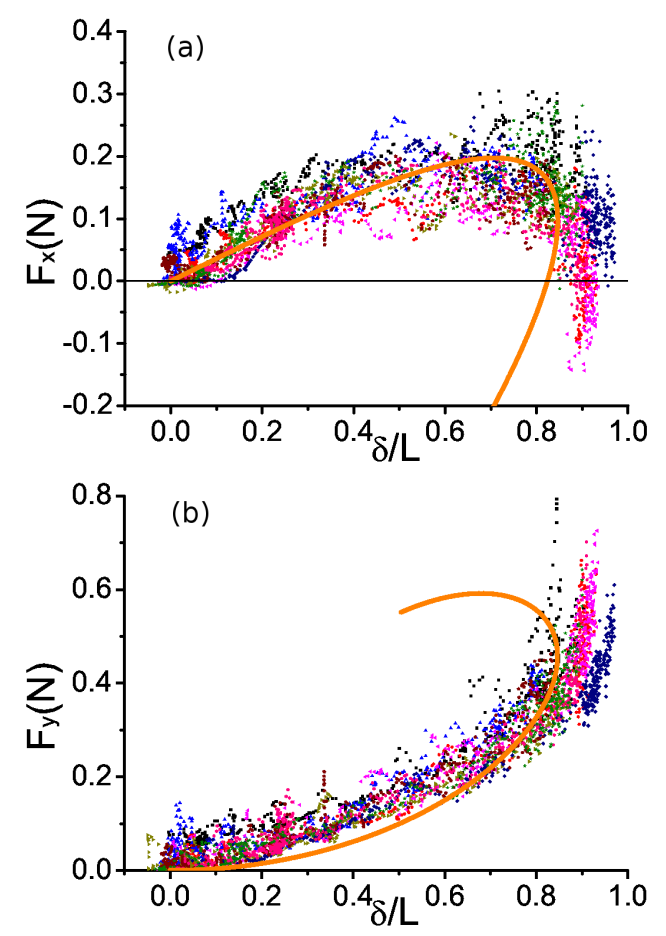

Figure 5. Superposition of the experimental forces for the 10 different experiments performed with the same control parameters $\left(L=3 \mathrm{~cm}, \phi_{0}=80.85 \%\right.$ and $\left.t=350 \mu \mathrm{m}\right)$ vs the lateral fiber deflection normalized by the fiber length $L$. Points of different colours correspond to the different experiments. The orange line is the simulation for an homogeneous orthogonal loading of the fiber. (a) For the cross-flow force $F_{x}$ (b) For the drag force $F_{y}$.
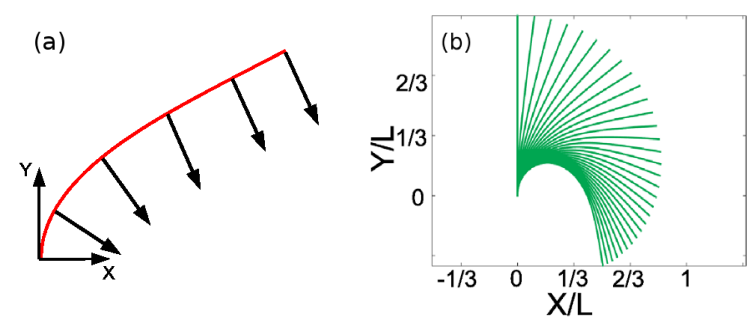

Figure 6. (a) Schematics of the homogeneous orthogonal loading of the fiber for Elastica simulations. (b) Superposition of the simulated fiber shapes for increasing forces.

\section{Conclusions}

We investigated the fluid/structure interaction between a flexible fiber and a dense granular flow close to the jamming transition. Above a critical fiber length, the fiber bends towards one side instead of oscillating around its initial straight position facing the granular flow. The critical length slightly decays with the 2D granular packing fraction in the investigated range of density ( $80 \%-83 \%)$.

In the case when the fiber bends, we showed that a simple model of homogeneous orthogonal loading produced by the grain flow against the fiber reasonably reproduces the experimental measurements of cross-flow and drag forces for intermediate fiber lengths.
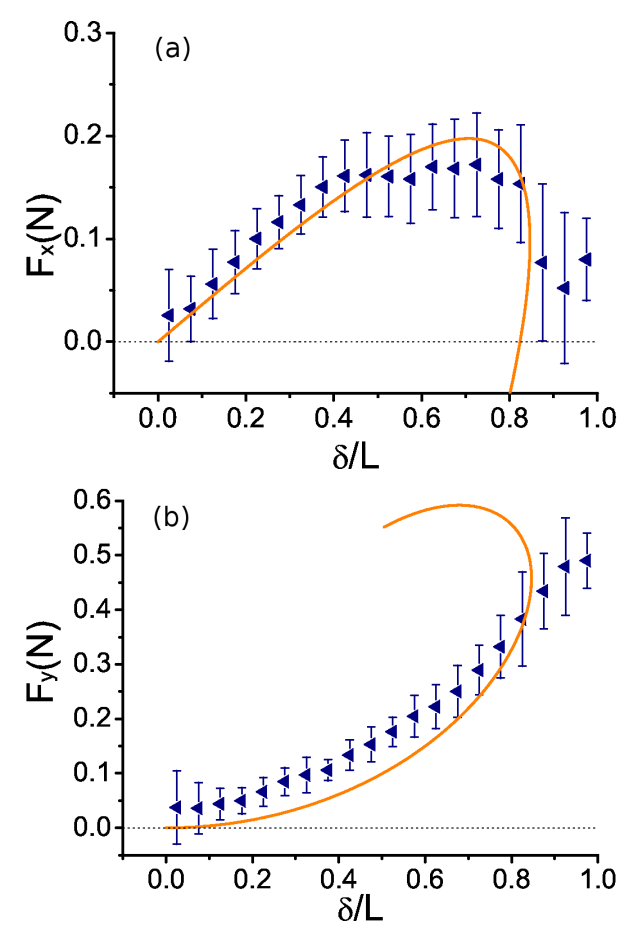

Figure 7. Comparison between the numerical results (lines) obtained for an homogeneous orthogonal loading of the fiber and the mean experimental forces (symbols). (a) Cross-flow force $F_{x}$ (b) Drag force $F_{y}$.

\section{Acknowledgements}

Thanks to N. Cheng, K. Luginbuhl, D. Wendell of A. Hosoi's group (MIT-France Seed Fund Project, Flexible Objects in Granular Media), Y. Takehara and F. Postic.

\section{References}

[1] L. Schouveiler, A. Boudaoud, J. Fluid Mech. 563, 71 (2006)

[2] F. Gosselin, E. de Langre, B.A. Machado-Almeida, J. Fluid Mech. 650, 319 (2010)

[3] S. Alben, M. Shelley, J. Zhang, Nature 420, 479 (2002)

[4] E. de Langre, Annu. Rev. Fluid Mech. 40, 141 (2008)

[5] S. Ramananarivo, R. Godoy-Diana, B. Thiria, Proc. Natl. Acad. Sci. 108, 5964 (2011)

[6] A.E. Hosoi, D.I. Goldman, Ann. Rev. Fluid Mech. 47, 431 (2015)

[7] K.M. Dorgan, P.A. Jumars, B. Johnson, B.P. Boudreau, E. Landis, Nature 433, 475 (2005)

[8] E. Lauga, T.R. Powers, Rep. Prog. Phys. 72 (2009)

[9] E. Kolb, P. Cixous, N. Gaudouen, T. Darnige, Phys. Rev. E 87, 032207 (2013)

[10] E. Kolb, P. Cixous, J.C. Charmet, Granular Matter 16, 223 (2014)

[11] A. Lazarus, C. Maurini, S. Neukirch, Stability of discretized nonlinear elastic systems (Springer Vienna, Vienna, 2015), pp. 1-53 